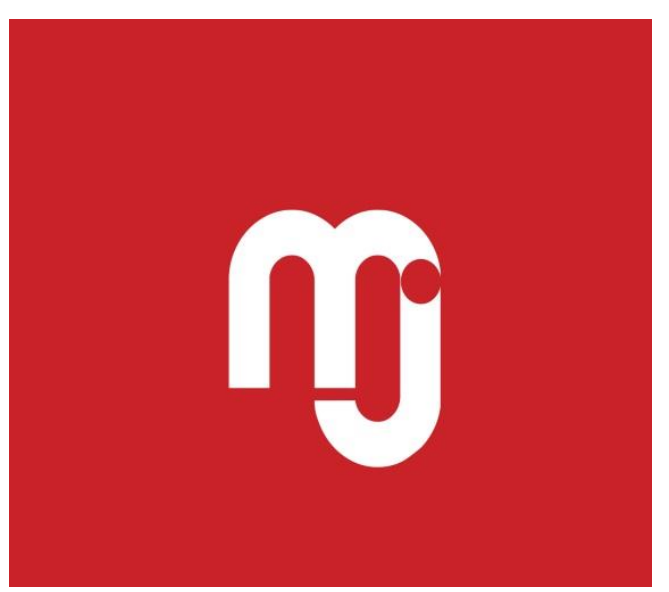

\title{
Metacritic Journal for Comparative Studies and Theory
}

Volume 7, Issue 1

July 2021 

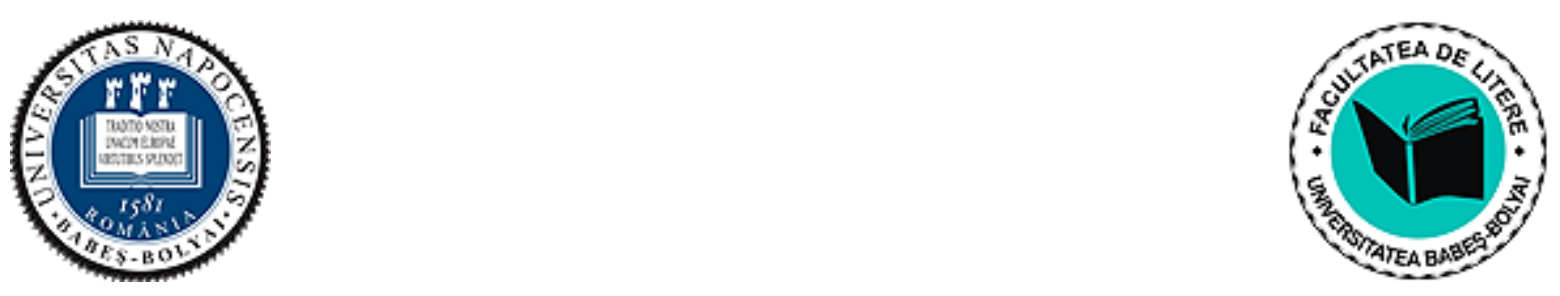

\begin{tabular}{|c|c|}
\hline \multicolumn{2}{|r|}{ Contact: } \\
\hline Support contact: & metacriticjournal@gmail.com \\
\hline Main contact: & $\begin{array}{l}\text { Mihaela Ursa, mihaela.ursa@ubbcluj.ro } \\
\text { Managing editor MJCST } \\
\text { Faculty of Letters, UBB } \\
\text { Horea str. 31, room } 211 \\
\text { 2002O2 - Cluj } \\
\text { Romania }\end{array}$ \\
\hline \multicolumn{2}{|r|}{ Editorial Team: } \\
\hline Editors-in-chief & Mihaela URSA, Alex GOLDIȘ \\
\hline Issue coordinators & Carmen BORBELY, Petronia POPA PETRAR, Daiana GÂRDAN \\
\hline Associate editors & Laura PAVEL, Alina PREDA, Adrian TUDURACHI, Maryam ZERBES \\
\hline Assistant editors & $\begin{array}{l}\text { Ana-Maria DELIU, Daiana GÂRDAN, Emanuel MODOC, Adrian } \\
\text { TĂTĂRAN }\end{array}$ \\
\hline Editorial board & Cristina DIAMANT, Gianina DRUȚĂ, Anamaria MIHĂILĂ, Ovio OLARU \\
\hline Advisory board & $\begin{array}{l}\text { Peter ARNDS (Trinity College, Dublin, Ireland) } \\
\text { Ioana BICAN (Babeș-Bolyai University, Cluj, Romania) } \\
\text { Philip FEDCHIN (St. Petersburg State University, Saint Petersburg, } \\
\text { Russia) } \\
\text { Till KUHNLE (University of Limoges, Limoges, France) } \\
\text { Liviu LUȚAŞ (Linnaeus University, Växjö, Sweden) } \\
\text { Norman MANEA (Bard College, New York, USA) } \\
\text { Călin-Andrei MIHĂILESCU (University of Western Ontario, London, } \\
\text { Canada) } \\
\text { Christian MORARU (University of North Carolina, Greensboro, USA) } \\
\text { Thomas PAVEL (University of Chicago, USA) } \\
\text { Doru POP (Babeş-Bolyai University, Cluj, România) } \\
\text { Mads ROSENDAHL THOMSEN (Aarhus University, Denmark) } \\
\text { Mihai SPĂRIOSU (University of Georgia, Athens, USA) } \\
\text { Monica SPIRIDON (University of Bucharest, Romania) } \\
\text { Bogdan ŞTEFĂNESCU (University of Bucharest, Bucharest, Romania) } \\
\text { Andrei TERIÄN (Lucian Blaga University, Sibiu, Romania) } \\
\text { Steven TÖTÖSY de ZEPETNEK (Academia Scientiarum et Artium } \\
\text { Europaea) } \\
\text { Olga VORONINA (Bard College, New York, USA) }\end{array}$ \\
\hline Copy editors & Mihnea BÂLICI, Maria CHIOREAN \\
\hline Proofreaders & $\begin{array}{l}\text { Zaher ALAJLANI, Ioana PAVEL, Alexandra TURCU, Krisztina KOCSIS, } \\
\text { Ana ȚĂRANU }\end{array}$ \\
\hline Layout editors & Eva SĂRĂȘAN, Adrian TĂTĂRAN \\
\hline
\end{tabular}

